\title{
Multi-UAV Cooperative Target Tracking via Consensus-based Guidance Vector Fields and Fuzzy MRAC
}

This paper was downloaded from TechRxiv (https://www.techrxiv.org).

\section{LICENSE}

CC BY 4.0

SUBMISSION DATE / POSTED DATE

07-01-2021 / 08-01-2021

\section{CITATION}

Muslimov, Tagir; Munasypov, Rustem (2021): Multi-UAV Cooperative Target Tracking via Consensus-based Guidance Vector Fields and Fuzzy MRAC. TechRxiv. Preprint. https://doi.org/10.36227/techrxiv.13537313.v1

$\mathrm{DOI}$ 


\title{
Multi-UAV Cooperative Target Tracking via Consensus- based Guidance Vector Fields and Fuzzy MRAC
}

\author{
Tagir Muslimov ${ }^{[0000-0002-9264-529 X]}$ and Rustem Munasypov \\ Ufa State Aviation Technical University, K. Marx Str., 12, 450008, Ufa, Russian Federation \\ tagir.muslimov@gmail.com
}

\begin{abstract}
This paper proposes a multi-agent approach to adaptive control of fixed-wing unmanned aerial vehicles (UAVs) tracking a moving ground target. The approach implies that the UAVs in a single group must maintain preset phase shift angles while rotating around the target so as to evaluate the target's movement more accurately. Thus, the controls should ensure that: (1) the UAV swarm follows a moving circular path whose center is the target while also attaining and maintaining a circular formation of a specific geometric shape; (2) the formation control systems is capable of self-tuning since the UAV dynamics is uncertain. In contrast to known studies, this one uses Lyapunov guidance vector fields that are direction- and magnitude-nonuniform. The overall cooperative controller structure is based on a decentralized and centralized consensus. This paper considers two interaction architectures: an open chain where each UAV only interacts with its neighbors; and cooperative leader, where the leading UAV is involved in attaining the formation. Using open chain decentralized architecture allows to have an unlimited number of aircraft in a group, which is in line with the swarm behavior concept. The cooperative controllers are self-tuned by fuzzy model reference adaptive control (MRAC). The approach was tested for efficiency and performance in various scenarios using complete nonlinear flyingwing UAV models equipped with configured standard autopilot models.
\end{abstract}

Keywords: Standoff Tracking, UAV Swarm Flocking, Formation Control, Cooperative Guidance, Collective Circumnavigation, Distributed Control, Decentralized Control

\section{Introduction}

Unmanned aerial vehicles (UAVs) have a wide range of applications. As many researchers note, using coordinated groups of autonomous mobile robots [1-3] including UAVs [4-7] can address a variety of complex problems that a single aircraft would not be able to solve. Besides, some standard autonomous fixed-wing UAV applications do benefit from using UAV groups; for instance, a tight formation consumes less resources [8]. Tracking a moving target is one problem that could be better solved by coordinated groups of autonomous fixed-wing UAVs $[9,10]$. In such scenarios, a UAV group must fly around the target following a circle or another kind of closed trajectory. On the other 
hand, aircraft in flight must attain and maintain preset distances between each other to enable more accurate assessment of the target's movement.

One approach to controlling a group of UAVs tracking a ground target is to follow a moving path [11]. If the target is the center of a circle, the tracking problem boils down to establishing this circular path and then following it strictly. Nevertheless, different strategies may apply when the UAV formation must be of a specific geometric shape. Kingston and Beard [12] proposed an approach based on a path following vector field that is nonuniform in direction only. Thus, they proposed to change the rotation radius so as to attain the required relative inter-UAV distance (and hence the phase shift angles when rotating around the target). However, Frew [9] notes that maintaining a constant rotation radius might take precedence over maintaining a constant flight speed. Another approach is to use speed controllers to attain the required inter-UAV distance. Thus, Frew et al. [13] studied controlling a two-UAV formation tracking a target. Summers et al. [14] used a different approach: they only enabled the speed controllers after the aircraft established a vector field, implying further strict retention of this field in flight.

This paper considers an approach to fixed-wing UAV group guidance for tracking a ground target; this approach is based on the author-devised method that uses directionand magnitude-nonuniform path following vector fields [15]. Unlike in known papers, the authors suggest that UAVs attain the required phase shift angles when they start to make a formation rather than after attaining a circular path. The global asymptotic stability of the control laws enables the method to perform well even if one or more UAVs digress.

Another distinctive feature of this approach is that UAVs are coordinated by decentralized consensus and interactive-leader consensus, where the leader is involved in attaining the formation. Thus, this group's interaction is be based on swarm behavior so that the formation could scale indefinitely [16]. Unlike in many other studies, the formation itself does not need equidistance, as the desired relative phase shift angles could be arbitrary. Many papers dwell upon decentralized UAV interaction; however, they made assumptions absent herein. Thus, Bukov et al. [17] studied a similar configuration with evidence from a dual-UAV leader-follower setup; Fathian et al. [18] ignored the input constraints in their proof of convergence. Linear consensus for UAVs was addressed by Kolaric et al. [19], Jia et al. [20], Liao et al. [21], etc.

When using a generalized autopilot-UAV system model to synthesize cooperative control laws, their further tuning becomes challenging. It is not only unmodeled dynamics, but also its uncertainty in flight that complicates the matter. Failure to fine-tune might result in destabilization and inability to attain the required formation. This is why this paper proposes using fuzzy model reference adaptive control (MRAC). Thus, the primary contribution of this research is that it implements adaptive self-tuning as part of an original consensus-based UAV formation control strategy for tracking a target. 


\section{$2 \quad$ Preliminary Notes and System Models}

\subsection{Assumptions}

Assumption 1. For space consideration, assume the target follows a rectilinear trajectory at a constant speed. The speed is assumed to be known in advanced. In practice, the speed could be metered onboard, e.g. by adaptive observers.

Assumption 2. Another assumption is that the weather is windless; however, the wind speed could be metered as well to adjust the guidance control laws.

Assumption 3. Each UAV is equipped with a standard preconfigured autopilot and is capable of calculating the distance to the UAVs it is interacting with. Besides, the UAVs in the group can detect the relative bearing with respect to the interacting UAVs.

\subsection{UAV model}

A real autopilot-UAV model is a fairly complex high-order system that features multiple nonlinearities. However, a high-level second-order unicycle model can be used to synthesize guidance control laws, as was done in many known studies, e.g. [22]:

$$
\dot{x}=v^{c} \sin \chi, \dot{y}=v^{c} \cos \chi, \dot{\chi}=a_{\chi}\left(\chi^{c}-\chi\right),
$$

where $x$ is the UAV coordinate on the eastward axis in an inertial coordinate system, $y$ is the UAV coordinate on the northward axis in the inertial coordinate system, $\chi$ is the current heading angle; $\chi^{c}$ is the heading angle control loop input; $v^{c}$ is the airspeed control loop input; $a_{\chi}$ is a positive constant that depends on the autopilot configuration and the UAV specifications.

Note that the numerical modeling for testing this approach will use complete nonlinear UAV models. Each UAV will be complemented with a configured autopilot model.

The model (1) implies input constraints that are inevitable in real-world autopilotUAV systems [22]:

$$
U \triangleq\left\{v^{c}, \dot{\chi}^{c} \mid 0<v_{\text {min }} \leq v^{c} \leq v_{\text {max }},-\dot{\chi}_{\text {max }} \leq \dot{\chi}^{c} \leq \dot{\chi}_{\text {max }}\right\} .
$$

To set a circular path that the UAV formation should establish when flying around their target, use the center $\mathbf{c}=\left(c_{e}(t), c_{n}(t), h(t)\right) \in \mathbb{R}^{3}$, the radius $\rho \in \mathbb{R}$, and the rotation direction $\lambda \in\{-1,1\}$ [23]:

$$
P_{o} \triangleq\left\{\mathbf{r} \in \mathbb{R}^{3}: \mathbf{r}=\mathbf{c}+\lambda \rho\left(\cos \varphi_{i}, \sin \varphi_{i}, 0\right)^{\mathrm{T}}, \varphi \in[0,2 \pi)\right\},
$$


where $c_{e}(t)$ is the center-of-circle coordinate on the eastward axis in an inertial coordinate system; $c_{n}(t)$ is the center-of-circle coordinate on the northward axis in an inertial coordinate system; $h(t)$ is the center-of-circle altitude (above the sea level); $\lambda=1$ defines clockwise movement, while $\lambda=-1$ defines counterclockwise movement; $\varphi_{i}$ is the current phase angle of the $i$ th aircraft. As the target is nonstationary, the coordinates of the center of a circular path are not constant and will change over time, unlike in [15]. Accordingly, the target speed $v_{\text {target }}$ is calculated as $v_{\text {target }}=\sqrt{c_{e}(t)^{2}+c_{n}(t)^{2}+h(t)^{2}}$.

\subsection{Multi-UAV System Model}

Graphs are normally used to describe the group interaction model. Many papers, e.g. [24] provide the standard notation.

This research uses an open-chain interaction topology similar to that in [15, 25]. In such cases, the system can be deemed decentralized as each UAV is only communicating with its neighbors.

Other interaction methods exist, e.g. interactive leader + followers [26]. This approach differs from the standard leader-follower method is that the leader is also involved in attaining and maintaining the formation.

An autonomous UAV formation can be analyzed as a multi-agent system consisting of $N$ autonomous agents, where $N \geq 2$. Let $\mathcal{N}_{i}$ be the set of all agents. Their interaction architecture can be described by a strongly connected graph as in [15, 25]:

$$
\mathcal{G} \triangleq(\mathcal{Q}, \mathcal{E})
$$

where the $i$ th UAV agent is the set of vertices in the graph $\eta_{i} \in \mathcal{Q}$, and each arc in the set $\mathcal{E}$ that leads from the vertex $\eta_{i}$ to the vertex $\eta_{j}$ means the agent $\eta_{i}$ receives data on the relative position of the agent $\eta_{j}$.

Thus, the set of arcs $\mathcal{E}$ shows the configured UAV interaction rules, which in case of an open chain are as follows [15]:

$$
\mathcal{E}=\{(1,2),(2,1),(2,3), \ldots,(N-1, N),(N, N-1)\} .
$$

When using an interactive leader + followers strategy, these rules can be written as

$$
\mathcal{E}=\{(1,2),(1,3),(1,4), \ldots,(1, N-1),(1, N)\} .
$$

Let $\mathcal{N}_{j}=\left(\eta_{j} \in \mathcal{Q}:\left(\eta_{i}, \eta_{j}\right) \in \mathcal{E}\right)$ be the set of the $i$ th agent's neighbors. 


\subsection{Decentralized and Centralized UAV Interaction Architecture}

This section describes a method for calculating the consensus-based control action vector; the method was also used in [15]. It was originally proposed in [27, 28] for mobile robots as linear agents. UAVs are expected to interact by the rules presented in (5) and (6).

Let $\mathbf{e}_{\theta} \in \mathbb{R}^{N \times 1}$ be this vector, where $\mathbb{R}^{N \times 1}$ is a space of $N \times 1$-dimensional matrices with components from $\mathbb{R}$. To find this vector, use some elements of the vector of all possible relative phase shift angle errors $\overline{\mathbf{e}}_{\theta}=\left(\hat{e}_{i, j}\right) \in \mathbb{R}^{N(N-1) \times 1}$, where $\hat{e}_{i, j}$ is the value of error for the directly interacting $i$ th and $j$ th agents. The choice is dictated by the interaction architecture; in this research, the control action vector is set as such for openchain interaction (5) similarly to [15] as

$$
\mathbf{e}_{\theta}=\left[\begin{array}{c}
e_{1} \\
\vdots \\
e_{k} \\
\vdots \\
e_{N}
\end{array}\right]=\left[\begin{array}{c}
\hat{e}_{12} \\
\vdots \\
-\hat{e}_{k-1, k}+\hat{e}_{k, k+1} \\
\vdots \\
-\hat{e}_{N-1, N}
\end{array}\right]=\hat{\mathbf{M}}_{\theta} \breve{\mathbf{e}}_{\theta}+\mathbf{D}
$$

and for interactive leader + followers $(6)$ as

$$
\mathbf{e}_{\theta}=\left[\begin{array}{c}
e_{1} \\
\vdots \\
e_{k} \\
\vdots \\
e_{N}
\end{array}\right]=\left[\begin{array}{c}
\hat{e}_{12}+\hat{e}_{13}+\ldots+\hat{e}_{1 N} \\
\vdots \\
-\hat{e}_{1, k} \\
\vdots \\
-\hat{e}_{1, N}
\end{array}\right]=\hat{\mathbf{M}}_{\theta} \breve{\mathbf{e}}_{\theta}+\mathbf{D}
$$

where $\mathbf{D}=-\mathbf{M}_{\theta} \mathbf{H}_{\theta}^{-1}\left(\left(\mathbf{P}_{\theta}^{d}\right)^{\mathrm{T}}, \hat{P}_{\theta}\right)^{\mathrm{T}}$ is a system control vector in the space of relative distances (a $(N-1)$-dimensional space generated by the interaction graph incidence matrix columns), $\mathbf{H}_{\theta}$ is a matrix that specifies the agents for agent-to-agent distance measurements, defined as follows:

$$
\mathbf{H}_{\theta}=\left[\begin{array}{c}
\mathbf{q}_{1} \\
\mathbf{q}_{2} \\
\vdots \\
\mathbf{q}_{N}
\end{array}\right], \quad \mathbf{q}_{i}=\left[\begin{array}{c}
\vdots \\
1 \\
\vdots \\
-1 \\
\vdots
\end{array}\right]^{\mathrm{T}}, i<N, \mathbf{q}_{N}=\left[\begin{array}{c}
1 \\
1 \\
\vdots \\
1
\end{array}\right]^{\mathrm{T}},
$$


whereby $\mathbf{H}_{\theta} \in \mathbb{R}^{N \times N}, \mathbf{q}_{i} \in \mathbb{R}^{1 \times N}$, positions of " 1 " and "-1" in $\mathbf{q}_{i}$ are determined according to the structure of the interaction graph;

$\mathbf{P}_{\theta}^{d} \in \mathbb{R}^{(N-1) \times 1}$ is the vector of desired inter-UAV phase shift angles, $\hat{P}_{\theta}=\sum_{k=1}^{N} \varphi_{k}$ is the total of the current UAV phase angles in an inertial coordinate system;

$\breve{\mathbf{e}}_{\theta}=\left(\breve{e}_{i, i+1}\right)_{i=\overline{1, N-1}} \in \mathbb{R}^{(N-1) \times 1}$ is the vector of current phase shift angles for directly coengaged agents, calculated by triple scalar product, e.g. when the final movement is directed clockwise:

if $\mathbf{n} \cdot\left(\mathbf{d}_{i} \times \mathbf{d}_{i+1}\right) \geq 0$, then $\breve{e}_{i, i+1}=\beta=\arccos \frac{\left(\mathbf{d}_{i}, \mathbf{d}_{i+1}\right)}{\left\|\mathbf{d}_{i}\right\|\left\|\mathbf{d}_{i+1}\right\|}$, and $\breve{e}_{i, i+1}=2 \pi-\beta$ in other cases, where $\mathbf{d}_{k}, k \in \mathcal{N}_{i}$ is the vector of aircraft-to-moving-target distance at a given time, $\mathbf{n}=(0,0,1)^{\mathrm{T}}$;

$\mathbf{M}_{\theta} \in \mathbb{R}^{N \times N}$ is an interaction matrix that in case of decentralized neighbor-neighbor interaction like herein is as follows:

$$
\mathbf{M}_{\theta}=\left[\begin{array}{ccccc}
-1 & 1 & 0 & \cdots & 0 \\
1 & -2 & \ddots & \ddots & \vdots \\
0 & \ddots & \ddots & 1 & 0 \\
\vdots & \ddots & 1 & -2 & 1 \\
0 & \cdots & 0 & 1 & -1
\end{array}\right] ;
$$

in case of an interactive leader + followers interaction, the matrix $\mathbf{M}_{\theta}$ must be presented as

$$
\mathbf{M}_{\theta}=\left[\begin{array}{ccccc}
-N & 1 & 1 & \cdots & 1 \\
1 & -1 & 0 & \cdots & 0 \\
1 & 0 & -1 & \ddots & \vdots \\
\vdots & \vdots & \ddots & \ddots & 0 \\
1 & 0 & \cdots & 0 & -1
\end{array}\right],
$$

in this case, the UAV number 1 serves as the interactive leader;

$\hat{\mathbf{M}}_{\theta} \in \mathbb{R}^{N \times(N-1)}$ is a matrix derived from the matrix $\mathbf{M}_{\theta} \mathbf{H}_{\theta}^{-1}$ by removing the $N$ th column. 


\section{Adaptive UAV Formation Control Strategy for Tracking a Moving Target}

The control must enable a UAV group coordinated as shown in the graph $\mathcal{G}$ (4) to fly in a circular orbit (3) at a given distance from the target (i.e. to perform standoff tracking) while also attaining and maintaining the preset phase shift angles. The formation must have prespecified parameters while it may be equidistant or of any other type.

\subsection{Nonuniform Vector Field for UAV Guidance in Standoff Tracking}

The control strategy is based on using a direction- and magnitude-nonuniform path following vector field $[15,25]$. To use this method, plot a vector field in each point of the UAV flight space to set the heading angle and the speed. However, the control laws obtained in [15] should be modified by adding the derivative signal as was done in [25]. Since direct differentiation can amplify noise and is undesirable, the derivative signal can be quite simply calculated analytically provided that the group members are communicating. In case of no communication, Kalman filtering can be applied to estimate the states. [25]:

The proposed modified UAV formation speed control law is thus written as follows

$$
\mathbf{v}^{c}=\left[\begin{array}{llll}
v_{1} & v_{2} & \ldots & v_{N}
\end{array}\right]^{\mathrm{T}}=v \mathbf{1}_{N}+\mathbf{L},
$$

where $\quad \mathbf{1}_{N}=\left[\begin{array}{llll}1 & 1 & \ldots & 1\end{array}\right]^{\mathrm{T}} \in \mathbb{R}^{N \times 1} \quad$ and the vector $\mathbf{L}=\left(v_{f}(2 / \pi) \arctan \left\{k_{\theta} e_{i}+k_{\dot{\theta}} \dot{e}_{i}\right\}\right)_{i=1, N} \in \mathbb{R}^{N \times 1}$ is found given (7) and (8), $k_{\theta}$ is the positive tuning coefficient, $k_{\dot{\theta}}$ is the positive tuning coefficient for the derivative signal, $v_{f}$ is the maximum norm of the additional velocity vector that is to be adjusted for the constraints (2), $v$ is the ultimate linear cruise speed of the UAVs provided that the target is stationary.

The following modified heading angle control law is proposed [25]:

$$
\chi^{c}=\left(\varphi_{i}+\lambda\left[\frac{\pi}{2}+\arctan \left(k_{o}\left(d_{i}-\rho\right)+k_{\dot{o}} \dot{d}_{i}\right)\right]\right)_{i=\overline{1, N}} \in \mathbb{R}^{N \times 1},
$$

where $d_{i}$ is the $i$ th UAV-to-target distance, $k_{o}$ is the tuning coefficient for the distanceto-circular-path signal, $k_{\dot{o}}$ is the tuning coefficient for the distance-to-circular-path derivative signal, and the remaining notation is as in (3). 


\subsection{Fuzzy Model Reference Adaptive Control for Self-Tuning in Target Tracking}

Finding the coefficients $k_{\theta}, k_{\dot{\theta}}, k_{o}$, and $k_{\dot{o}}$ for the equations (9) and (10) is a complex problem, as the UAV dynamics may change over time for various reasons and is inherently uncertain. However, incorrect coefficients might effectively prevent the group from establishing an accurate formation on a given path or even cause the group to lose stability.

This section describes how fuzzy model reference adaptive control could enable selftuning for these coefficients. A similar approach was used in [29] to self-tune the parameters for a UAV group following a rectilinear path. The strategy essentially boils to adding the second-order unicycle model (1) with input constraints (2) to each UAV, which will use the data from other UAVs to calculate the output. Each of these models needs fine-tuned coefficients $a_{\chi}$. Collectively, the decentralized unicycle model of each UAV is the reference model. This reference model should be tuned in advance to acceptable control quality, which can be done, for instance, empirically. Then each UAV engages its fuzzy tuning controller to tune the coefficients by applying the error signal, where the error is found by comparing the reference model output to the actual relative positions in the group. The fuzzy controller also uses this error's derivative signal, which, once again, can be computed analytically. Figure 1 shows the general flowchart of the proposed self-tuning approach.

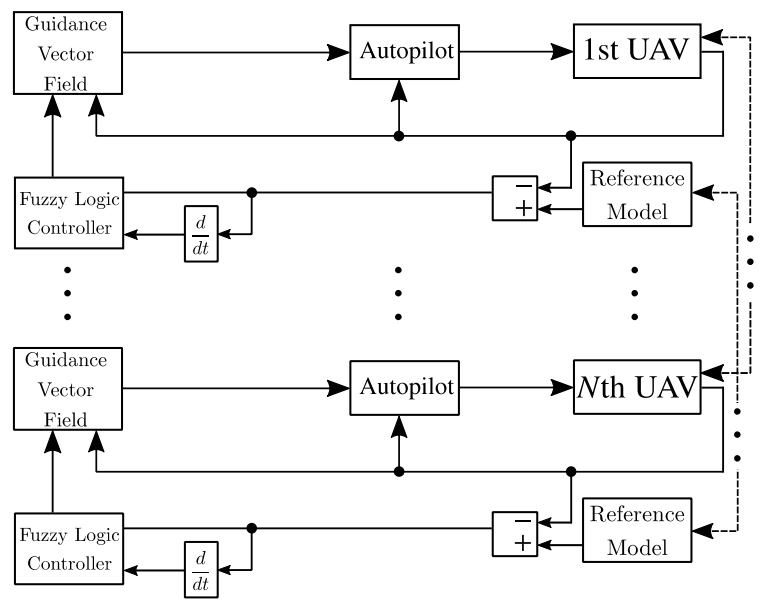

Fig. 1. Fuzzy model reference adaptive control for a UAV formation

Rules for the fuzzy controller are chosen on the basis of the fuzzy Lyapunov functions [30]. Unlike in [29], the authors' approach implies making rules for the error derivative signal as well. Another difference is that the authors' approach involves tuning not only for the distance-to-final-path error coefficient, but also for the derivative of the same error. 
Let us use the following notation: $y=\hat{d}_{i}-\hat{d}_{i}^{m}$, where $\hat{d}_{i}=d_{i}-\rho$ is the error of the $i$ th UAV's distance to the circular path; $\hat{d}_{i}^{m}=d_{i}^{m}-\rho$ is the error of the $i$ th UAV's reference model distance to the circular path; $\Delta k_{o}$ and $\Delta k_{\dot{o}}$ are additional coefficients described below; $W=\Delta k_{o} \hat{d}_{i}+\Delta k_{\dot{j}} \dot{d}_{i}$ is an additional term added to the control laws to tune the parameters. The resulting control law (10) complete with adaptive tuning is written as follows:

$$
\chi^{c}=\left(\varphi_{i}+\lambda\left[\frac{\pi}{2}+\arctan \left(\left(k_{o}+\Delta k_{o}\right)\left(d_{i}-\rho\right)+\left(k_{\dot{o}}+\Delta k_{\dot{o}}\right) \dot{d}_{i}\right)\right]\right)_{i=\overline{1, N}} .
$$

Then, according to [29] the following fuzzy rules can apply:

IF $y$ is positive AND $\dot{y}$ is positive THEN $W$ is negative big

IF $y$ is negative AND $\dot{y}$ is negative THEN $W$ is positive big

IF $y$ is positive AND $\dot{y}$ is negative THEN $W$ is zero

IF $y$ is negative AND $\dot{y}$ is positive THEN $W$ is zero

IF $y$ is zero AND $\dot{y}$ is positive THEN $W$ is negative

IF $y$ is zero AND $\dot{y}$ is negative THEN $W$ is positive

Similar reasoning applies when tuning the parameters for speed control laws (9).

The authors hereof used standard triangular, trapezoidal-shaped membership functions, a center-of-gravity defuzzifier, and the product inference engine to produce a Mamdani controller. Figure 2a to $2 \mathrm{~b}$ show the selected membership functions for $y$, and $\Delta k_{o}$, respectively. Similar membership functions can be chosen for $\dot{y}, \Delta k_{\dot{o}}$, and tuning the UAV speed control law.

(a)

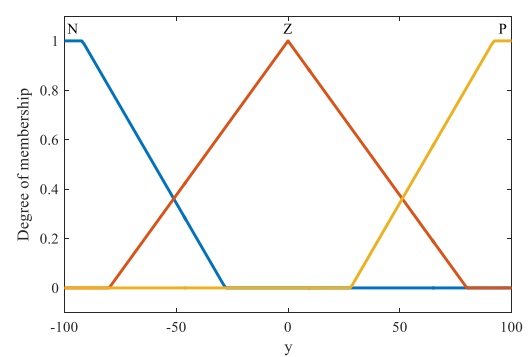

(b)

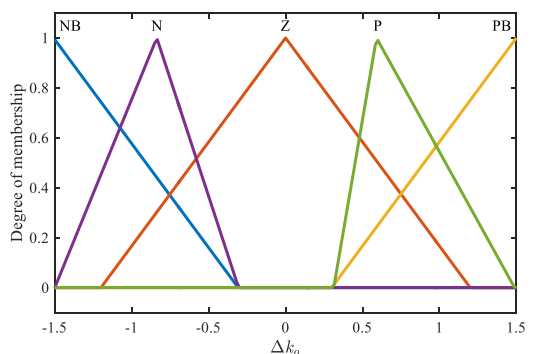

Fig. 2. Membership functions for the fuzzy controller. (a) Membership functions for the path error $y$ input. (b) Membership functions for the coefficient $\Delta k_{o}$ output. 


\section{Output of Numerical Simulation on Complete UAV Models}

\subsection{Model used for numerical tests; modeling parameters}

For numerical tests of the proposed approach, the researchers used complete nonlinear models of Zagi fixed-wing UAVs with 6 degrees of freedom and 12 states. Each UAV model was equipped with a standard tuned autopilot synthesized by successive loop closure. For UAV specifications, see [23].

Table 1 shows the simulation parameters that were used in all modeling scenarios to test and assess the performance of the proposed control strategy. Each UAV autopilot was also tasked to keep a 100-meter altitude.

Table 1. Simulation parameters

\begin{tabular}{lccc}
\hline \multicolumn{1}{c}{ Parameter } & Symbol & Values \\
\hline Initial UAV heading vector, [rad] & $\chi(0)$ & {$\left[\begin{array}{llll}0 & 0 & 0 & 0\end{array}\right]^{\mathrm{T}}$} \\
Initial UAV speed vector, [m/s] & $\mathbf{v}(0)$ & {$\left[\begin{array}{cccc}13 & 13 & 13 & 13\end{array}\right]^{\mathrm{T}}$} \\
Circular path radius, [m] & $\rho$ & 200 & \\
Initial target coordinates, [m] & $\mathbf{c}=\left[\begin{array}{cccc}c_{e}(0) & c_{n}(0) & h(0)\end{array}\right]^{\mathrm{T}}$ & {$\left[\begin{array}{lll}500 & 500 & 100\end{array}\right]^{\mathrm{T}}$} \\
Ultimate cruise speed of the formation & $v$ & 13 & \\
in case of a stationary target [m/s] & $v_{\text {target }}$ & 2 & $\pi / 4$ \\
Target speed, [m/s] & $\chi_{\text {target }}$ & $\mathbf{p}_{1}(0)$, & {$\left[\begin{array}{lll}380 & 630 & -100\end{array}\right]^{\mathrm{T}}$,} \\
Target course angle, [rad] & $\mathbf{p}_{2}(0)$, & {$\left[\begin{array}{lll}475 & 450 & -100\end{array}\right]^{\mathrm{T}}$,} \\
& $\mathbf{p}_{3}(0)$, & {$\left[\begin{array}{lll}330 & 375 & -100\end{array}\right]^{\mathrm{T}}$,} \\
Vector of initial UAV coordinates & $\mathbf{p}_{4}(0)$ & {$\left[\begin{array}{lll}710 & 395 & -100\end{array}\right]^{\mathrm{T}}$} \\
in the ICS, [m] & $\mathbf{P}_{\theta}^{d}$ & {$\left[\begin{array}{lll}270 & 260 & 290\end{array}\right]^{\mathrm{T}}$} \\
& $k_{\theta}, k_{\dot{\theta}}, k_{o}, k_{\dot{o}}$ & $40,35,1,4$
\end{tabular}

\subsection{Simulation Results}

For space considerations, this section presents the results of testing the adaptive selftuning for the heading angle control laws. Similar results can be obtained for speed controls, too. Simulation used two scenarios; in Scenario 1, the selected coefficients differed significantly from those obtained for the reference model. Table 1 specifies the coefficients. In Scenario 2, the parameters were the same, but model reference adaptive 
control was involved. Each scenario was tested with two interaction architectures: open chain and interactive leader + followers.

The researchers first ran simulations for the open-chain topology. Figure $3 \mathrm{a}$ shows the temporal characteristics for the path errors of each UAV with untuned coefficients (Scenario 1). Figure $3 b$ illustrates transient trajectories for this case.

(a)

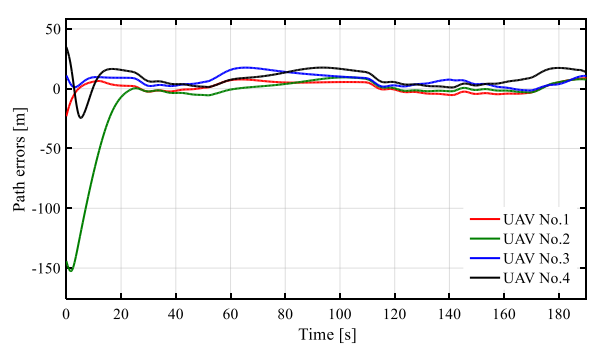

(b)

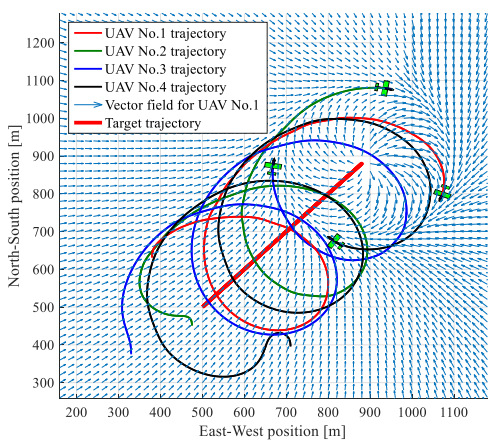

Fig. 3. UAV formation control for target tracking. Untuned coefficients, open-chain interaction. (a) Temporal characteristics of the UAV path errors. (b) Transient trajectories of the UAVs when attaining and maintaining the formation at the time $t=190 \mathrm{~s}$. The figure also shows the path following vector field for the UAV \#1

Then, the same open-chain topology was tested again with the same untuned coefficients, but this time using fuzzy MRAC-based self-tuning. Figure 4a shows the temporal characteristics of the path errors for each UAV in the self-tuning Scenario 2. Figure $4 \mathrm{~b}$ illustrates the transient trajectories for this scenario.

(a)

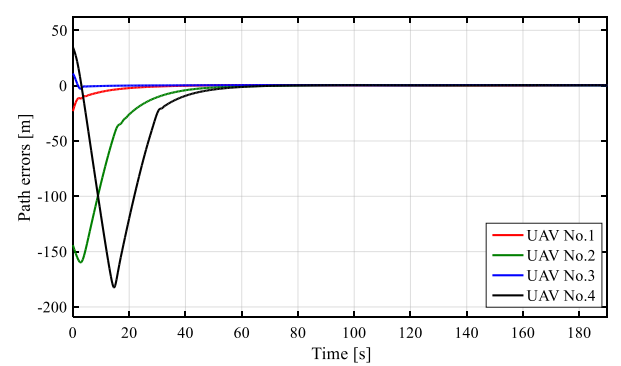

(b)

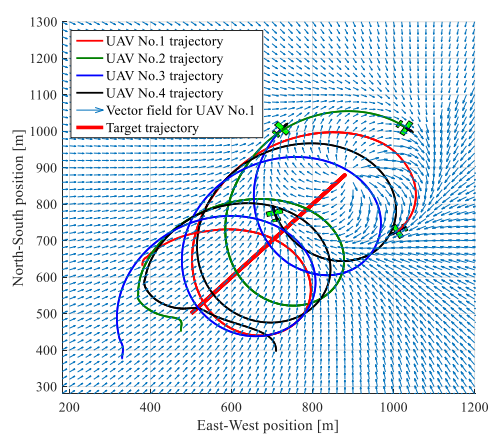

Fig. 4. UAV formation control for target tracking. Fuzzy MRAC-based self-tuning, open-chain interaction. (a) Temporal characteristics of the UAV path errors. (b) Transient trajectories of the UAVs when attaining and maintaining the formation at the time $t=190 \mathrm{~s}$. The figure also shows the path following vector field for the UAV \#1 
Figures $5 \mathrm{a}$ and $5 \mathrm{~b}$ show how the coefficients $\Delta k_{o}$ and $\Delta k_{\dot{o}}$ changed.

(a)

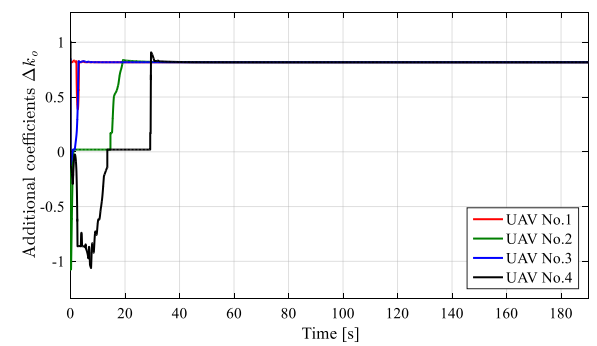

(b)

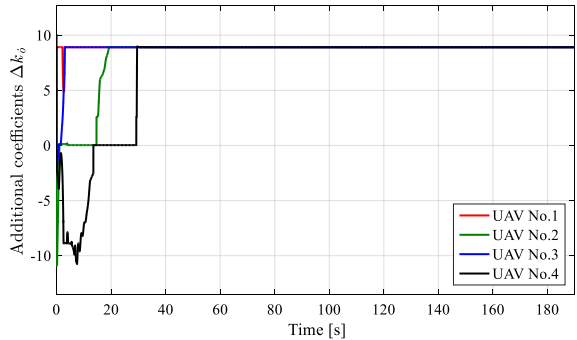

Fig. 5. UAV formation control for target tracking. Fuzzy MRAC-based self-tuning, open-chain interaction. (a) Temporal characteristics for the coefficients $\Delta k_{o}$. (b) Temporal characteristics for the coefficients $\Delta k_{\dot{o}}$.

Then the authors ran simulations for the interactive leader + followers topology. Figure 6a shows the temporal characteristics for the path errors of each UAV with untuned coefficients (Scenario 1). Figure 6b illustrates transient trajectories for this case.

(a)

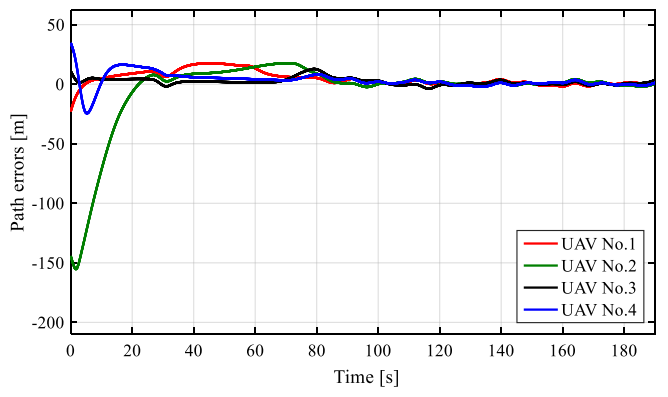

(b)

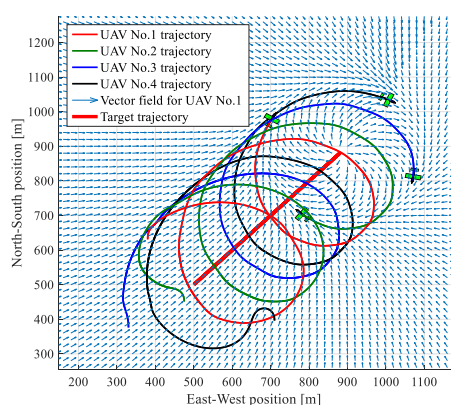

Fig. 6. UAV formation control for target tracking. Untuned coefficients, interactive leader + followers interaction. (a) Temporal characteristics of the UAV path errors. (b) Transient trajectories of the UAVs when attaining and maintaining the formation at the time $t=190 \mathrm{~s}$. The figure also shows the path following vector field for the UAV \#1.

Then, the same interactive leader + followers topology was tested again with the same untuned coefficients, but this time using fuzzy MRAC-based self-tuning. Figure 7 a shows the temporal characteristics of the path errors for each UAV in the self-tuning Scenario 2. Figure $7 \mathrm{~b}$ illustrates the transient trajectories for this scenario. 
(a)

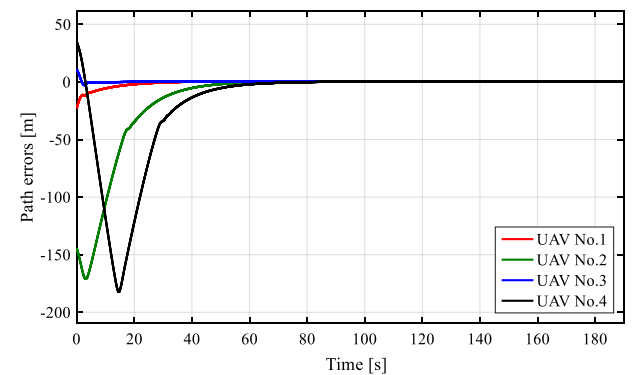

(b)

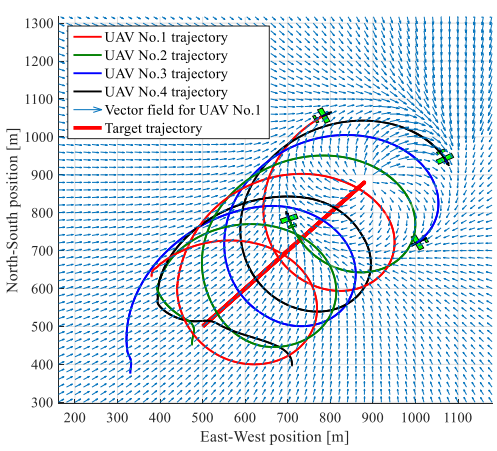

Fig. 7. UAV formation control for target tracking. Fuzzy MRAC-based self-tuning, interactive leader + followers interaction. (a) Temporal characteristics of the UAV path errors. (b) Transient trajectories of the UAVs when attaining and maintaining the formation at the time $t=190 \mathrm{~s}$. The figure also shows the path following vector field for the UAV \#1.

Apparently, the simulation showed such adaptive self-tuning not only to help preserve stability but also to improve the quality of transient trajectories. Also note that the nonconvergence of path errors in case of untuned coefficients effectively prevents accurate formation attainment, i.e. the phase shift angles in the UAV group will not converge. Thus, fuzzy model reference adaptive control enables UAV formation control to self-tune even in case of uncertain aircraft dynamics, which is inevitable in realworld autopilot-UAV systems.

\section{Conclusions}

This paper proposes a UAV formation control approach for tracking a moving target. The control strategy is based on the authors' method that was developed earlier and uses magnitude- and direction-nonuniform path following vector fields. Given the high oscillations of the control loop processes, the control laws for stationary targets were modified by adding derivative signals. However, the quality and stability of attaining and maintaining a UAV formation greatly depends on the coefficient tuning; finding the optimal coefficients might be difficult due to the uncertain and changing UAV dynamics. For this reason, the authors propose using fuzzy model reference adaptive control to enable parameter self-tuning. Detailed simulation using complete nonlinear UAV models showed such adaptive self-tuning capable and efficient for decentralized and centralized UAV group interaction architectures.

Acknowledgements The reported study was funded by RFBR according to the research project № 18-08-01299. 


\section{References}

1. Ivanov, D.: Decentralized Planning of Intelligent Mobile Robot's Behavior in a Group with Limited Communications. In: Advances in Intelligent Systems and Computing. pp. 418-427. Springer Verlag (2019). https://doi.org/10.1007/978-3-030-01821-4_44.

2. Darintsev, O.V., Yudintsev, B.S., Alekseev, A.Y., Bogdanov, D.R., Migranov, A.B.: Methods of a Heterogeneous Multi-agent Robotic System Group Control. Proc. 13th Int. Symp. "Intelligent Syst. 2018." 150, 687-694 (2019). https://doi.org/10.1016/j.procs.2019.02.032.

3. Zenkevich, S.L., Nazarova, A. V., Huo, J.: Formation Control of Ground Multi-agent System Using Quadcopter with Camera. In: Studies in Systems, Decision and Control. pp. 113-124. Springer (2020). https://doi.org/10.1007/978-3-030-37841-7_9.

4. Alonso-Mora, J., Montijano, E., Nägeli, T., Hilliges, O., Schwager, M., Rus, D.: Distributed multi-robot formation control in dynamic environments. Auton. Robots. 43, 1079-1100 (2019). https://doi.org/10.1007/s10514-018-9783-9.

5. Milyakov, D.A., Merkulov, V.I.: The Approach to Managing a Group of UAVs as a System with Distributed Parameters. Proc. 13th Int. Symp. "Intelligent Syst. 2018." 150, 39-45 (2019). https://doi.org/10.1016/j.procs.2019.02.009.

6. Hock, A., Schoellig, A.P.: Distributed iterative learning control for multi-agent systems: Theoretic developments and application to formation flying. Auton. Robots. 43, 19892010 (2019). https://doi.org/10.1007/s10514-019-09845-4.

7. Guzey, H.M., Dierks, T., Jagannathan, S., Acar, L.: Modified Consensus-based Output Feedback Control of Quadrotor UAV Formations Using Neural Networks. J. Intell. Robot. Syst. 94, 283-300 (2019). https://doi.org/10.1007/s10846-018-0961-y.

8. Zhang, Q., Liu, H.H.T.: Aerodynamic model-based robust adaptive control for close formation flight. Aerosp. Sci. Technol. 79, 5-16 (2018). https://doi.org/10.1016/j.ast.2018.05.029.

9. Frew, E.W.: Sensitivity of cooperative target geolocalization to orbit coordination. J. Guid. Control. Dyn. 31, 1028-1040 (2008). https://doi.org/10.2514/1.32810.

10. Kokolakis, N.-M.T., Koussoulas, N.T.: Coordinated Standoff Tracking of a Ground Moving Target and the Phase Separation Problem. In: 2018 International Conference on Unmanned Aircraft Systems, ICUAS 2018. pp. 473-482. (2018). https://doi.org/10.1109/ICUAS.2018.8453292.

11. Wang, Y., Wang, D., Zhu, S.: Cooperative moving path following for multiple fixedwing unmanned aerial vehicles with speed constraints. Automatica. 100, 82-89 (2019). https://doi.org/10.1016/j.automatica.2018.11.004.

12. Kingston, D., Beard, R.W.: UAV splay state configuration for moving targets in wind. In: Advances in Cooperative Control and Optimization. pp. 109-128. Springer, Berlin (2007).

13. Frew, E.W., Lawrence, D.A., Morris, S.: Coordinated standoff tracking of moving targets using Lyapunov guidance vector fields. J. Guid. Control. Dyn. 31, 290-306 (2008).

14. Summers, T.H., Akella, M.R., Mears, M.J.: Coordinated standoff tracking of moving targets: Control laws and information architectures. J. Guid. Control. Dyn. 32, 56-69 (2009).

15. Muslimov, T.Z., Munasypov, R.A.: Consensus-Based Cooperative Circular Formation Control Strategy for Multi-UAV System. In: International Russian Automation 
Conference (RusAutoCon). pp. 1-8. IEEE $\quad$ (2019). https://doi.org/10.1109/RUSAUTOCON.2019.8867733.

16. Hamann, H.: Introduction to Swarm Robotics. In: Swarm Robotics: A Formal Approach. pp. 1-32. Springer International Publishing, Cham (2018). https://doi.org/10.1007/978-3-319-74528-2_1.

17. Bukov, V., Bronnikov, A., Selvesyuk, N.: Decentralized coordinated control for a group of aircraft. Proc. Inst. Mech. Eng. Part G J. Aerosp. Eng. 224, 647-655 (2010). https://doi.org/10.1243/09544100JAERO671.

18. Fathian, K., Summers, T.H., Gans, N.R.: Distributed Formation Control and Navigation of Fixed-wing UAVs at Constant Altitude. In: 2018 International Conference on Unmanned Aircraft Systems (ICUAS). pp. 300-307. IEEE (2018). https://doi.org/10.1109/ICUAS.2018.8453462.

19. Kolaric, P., Chen, C., Dalal, A., Lewis, F.L.: Consensus controller for multi-UAV navigation. Control Theory Technol. 16, 110-121 (2018). https://doi.org/10.1007/s11768-018-8013-5.

20. Jia, Y., Li, Q., Zhang, W.: A distributed cooperative approach for unmanned aerial vehicle flocking. Chaos An Interdiscip. J. Nonlinear Sci. 29, 043118 (2019). https://doi.org/10.1063/1.5082574.

21. Liao, W., Wei, X., Lai, J., Sun, H.: Formation control for multi-UAVs systems based on Kullback-Leibler divergence. Trans. Inst. Meas. Control. 42, 598-603 (2020). https://doi.org/10.1177/0142331219878581.

22. Ren, W., Beard, R.W.: Trajectory Tracking for Unmanned Air Vehicles With Velocity and Heading Rate Constraints. IEEE Trans. Control Syst. Technol. 12, 707-716 (2004).

23. Beard, R.W., McLain, T.W.: Small unmanned aircraft: Theory and practice. Princeton University Press (2012).

24. Ahn, H.-S.: Preliminary Background. In: Formation Control. Studies in Systems, Decision and Control. pp. 3-26. Springer, Cham (2020). https://doi.org/10.1007/978-3030-15187-4_1.

25. Muslimov, T.Z., Munasypov, R.A.: Coordinated UAV Standoff Tracking of Moving Target based on Lyapunov Vector Fields. 2020 Int. Conf. Nonlinearity, Information and Robotics (NIR), Innopolis, $\quad$ Russia, $\quad 3-6 \quad$ Dec. 2020. https://doi.org/10.1109/NIR50484.2020.9290189

26. Hou, Z., Fantoni, I.: Interactive Leader-Follower Consensus of Multiple Quadrotors Based on Composite Nonlinear Feedback Control. IEEE Trans. Control Syst. Technol. 26, 1732-1743 (2018). https://doi.org/10.1109/TCST.2017.2738602.

27. Yamaguchi, H., Arai, T., Beni, G.: A distributed control scheme for multiple robotic vehicles to make group formations. Rob. Auton. Syst. 36, 125-147 (2001). .

28. Yamaguchi, H., Arai, T.: Distributed and autonomous control method for generating shape of multiple mobile robot group. Proc. IEEE/RSJ Int. Conf. Intell. Robot. Syst. 2, 800-807 (1994).

29. Muslimov, T.Z., Munasypov, R.A.: UAV Formation Flight Using Non-Uniform Vector Field and Fuzzy Self-Tuning PD-Control. 2018 Int. Russ. Autom. Conf. 1-6 (2018). https://doi.org/10.1109/RUSAUTOCON.2018.8501769.

30. Margaliot, M., Langholz, G.: New approaches to fuzzy modeling and control: design and analysis. World Scientific, Singapore, New Jersey, London, Hong Kong (2000). 